\title{
Erratum: Conserved and host-specific features of influenza virion architecture
}

\author{
Edward C. Hutchinson, Philip D. Charles, Svenja S. Hester, Benjamin Thomas, David Trudgian, \\ Mónica Martínez-Alonso \& Ervin Fodor
}

Nature Communications 5:4816 doi: 10.1038/ncomms5816 (2014); Published 16 Sep 2014; Updated 17 Feb 2015

In the last paragraph of the Methods section in this Article, the dates on which certain data were downloaded from public databases were typeset incorrectly. The following are the correct details: 'A list of proteins identified in exosomes was downloaded from www.exocarta.org on 12 March 2014 (refs 39,40). A hypergeometric test was used to calculate the probability that our samples were enriched with these exosomal markers by chance: after removing redundancies, the number of occurrences of markers in the data in Supplementary Data 3-5 (705/907 proteins) was compared with the total number of markers $(3,442)$ and the number of proteins annotated by EBI on 12 June 2014 in the genomes of humans, cows and chickens $(20,649,19,634$ and 15,621, respectively)'. 